\title{
Torque Measurement of 3-DOF Haptic Master Operated by Controllable Electrorheological Fluid
}

\author{
Jong-Seok $\mathrm{Oh}^{1}$, Seung-Bok Choi ${ }^{1}$ and Yang-Sub Lee ${ }^{2}$ \\ ${ }^{1}$ Department of Mechanical Engineering, Inha University, Incheon 402-751, Korea \\ ${ }^{2}$ Faculty of Mechanical and Automotive Engineering, Keimyung University, Daegu, 704-701, Korea \\ ojshero@gmail.com; seungbok@inha.ac.kr, (corresponding author); lysgd@kmu.ac.kr
}

\begin{abstract}
This work presents a torque measurement method of 3-degree-of-freedom (3-DOF) haptic master featuring controllable electrorheological (ER) fluid. In order to reflect the sense of an organ for a surgeon, the ER haptic master which can generate the repulsive torque of an organ is utilized as a remote controller for a surgery robot. Since accurate representation of organ feeling is essential for the success of the robot-assisted surgery, it is indispensable to develop a proper torque measurement method of 3DOF ER haptic master. After describing the structural configuration of the haptic master, the torque models of ER spherical joint are mathematically derived based on the Bingham model of ER fluid. A new type of haptic device which has pitching, rolling, and yawing motions is then designed and manufactured using a spherical joint mechanism. Subsequently, the field-dependent parameters of the Bingham model are identified and generating repulsive torque according to applied electric field is measured. In addition, in order to verify the effectiveness of the proposed torque model, a comparative work between simulated and measured torques is undertaken.
\end{abstract}

Keywords: Torque measurement, haptic master, repulsive torque, Bingham model, torque model.

\section{INTRODUCTION}

$\mathrm{I}^{\mathrm{T}}$ T IS GENERALLY OBSERVED that the yield stress of electrorheological (ER) fluid significantly increases when subjected to external electric fields [1]. Since the yield stress of ER fluid can be continuously controlled according to the control electric input, semi-active damping control systems featuring ER fluid are very attractive for many application systems. It is also known that other advantages of ER applications include fast response time, quiet operation, simple mechanism, low power consumption, and high stability [2]. Due to these advantages, tremendous researches such as damper [3, 4], and brake [5, 6] have been triggered. Particularly, previous studies related to ER brake were utilized to keep from transmitting actuating torque of motor in automotive field. Since the ER brake only needs to generate 1-DOF motion, the brake mechanism is very simple. Accordingly, experimental methods of ER brake for torque measurement and tracking control are relatively easy. However, braking mechanism has recently been adopted to realize the repulsive force along the motions of a surgeon in medical fields. Therefore, there have been continual calls for a new kind of brake mechanism which can realize the multi motions of a surgeon.

Typically, it is known that a robot-assisted surgery needs support of a master-slave robot system. The master commands the slave robot to perform surgery. In order to reflect the physical constraints of the robot to the surgeon, the realization of human sense such as haptic master has been broadly researched in robotic fields. Most of the haptic master devices had utilizing motors, links or wires. While these systems can generally reflect the reaction force of an organ, they also have problems such as complex mechanism and safety problem concerning unexpected movement of actuators [7]. Moreover, it is difficult to control reaction force continuously and smoothly. Therefore, several researches featuring ER fluid have been investigated for the haptic master system. Kikuchi et al. developed a new practical haptic device 'PLEMO-P1'; this system adopted ER brakes as its force generators [8]. Furusho et al. developed an ER brake and showed a passive force display system using ER brakes with two degrees of freedom [9]. However, these researches were conducted by using 1-DOF ER brake. Therefore, researches utilizing multi-DOF ER brake are required to realize the multi motions of a surgeon. In addition, it is indispensable to develop the experimental methods of multi-DOF ER brake for torque measurement.

Consequently, the main contribution of this work is to propose a torque measurement method of 3-degree-offreedom (3-DOF) haptic master featuring controllable ER fluid. Due to spherical joint mechanism, the proposed haptic master is not only to generate three rotational motions but also to reflect the reaction force of an organ. In addition, by establishing the proper torque measurement method, it is possible to measure repulsive torque of the master device accurately. The torque models along rotational motions are derived based on the Bingham model and the geometric features of the ER haptic master. After obtaining the fielddependent value of the Bingham model, the torque measurement test is conducted to compare predicted torque. It is demonstrated via experimental investigation that the predicted torque value is successfully achieved by implementing the proposed torque measurement method which is specially set for the 3-DOF haptic master. It is also shown that the proposed haptic master can generate appropriate torque level required for robot-assisted surgery application. 


\section{MODELING OF HAPTIC MASTER}

A spherical joint featuring ER fluid has been proposed to achieve repulsive force-feedback along 3-DOF rotational motions. The repulsive force-feedback is realized by ER fluid which behaves as the Bingham model whose constitutive equation is given by [10]:

$$
\begin{gathered}
\tau=\eta \dot{\gamma}+\tau_{y}(E) \\
\tau_{y}(E)=\alpha E^{\beta}
\end{gathered}
$$

where $\tau$ is the shear stress, $\eta$ is the viscosity of ER fluid, $\dot{\gamma}$ is the shear rate, and $\tau_{y}(E)$ is the field-dependent dynamic yield stress of the ER fluid. Dynamic yield stress exponentially increases according to the electric field, $E$. The Bingham model of ER fluid is utilized to derive the torque model of ER spherical joint [11].

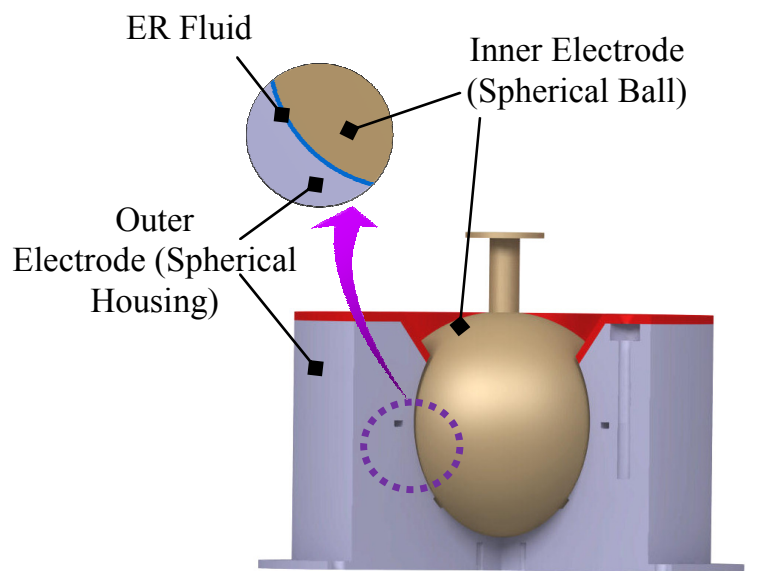

Fig.1. Schematic configuration of ER haptic master.

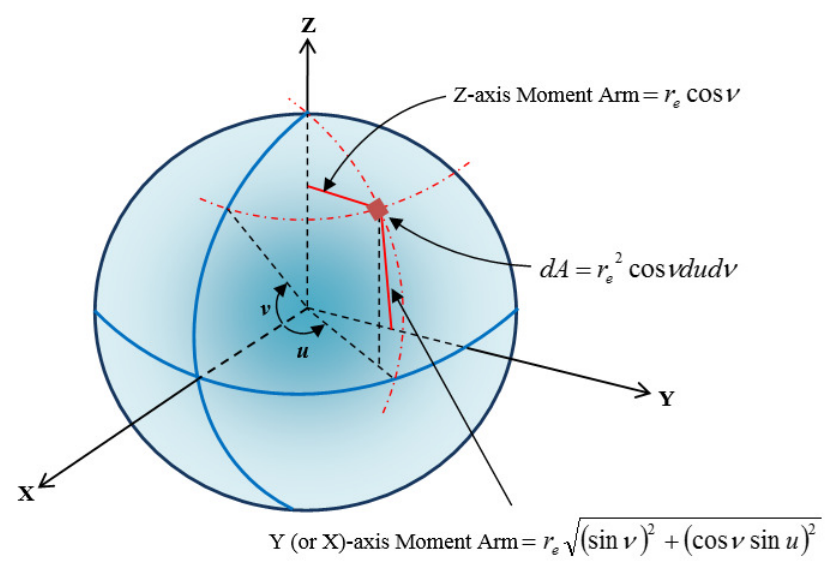

Fig.2. Coordinate system for spherical joint mechanism.

Fig.1. shows the schematic configuration of the ER spherical joint which consists of a spherical ball and spherical housing. The ER fluid is inserted between the spherical ball and spherical housing. In order to apply electric field to ER fluid, the role of spherical ball and spherical housing is inner and outer electrodes, respectively. A tape which has a very small friction coefficient is attached to maintain gap distance at 6 points of spherical housing. The torque induced by ER spherical joint can be expressed as follows:

$$
T_{\text {total }}=T_{c}+T_{\eta}+T_{f}
$$

where $T_{c}$ is the torque induced by dynamic yield stress of ER fluid, $T_{\eta}$ is the friction torque induced by ER fluid and $T_{f}$ is mechanical friction torque. $T_{c}$ and $T_{\eta}$ can be derived by using (1)

$$
\begin{aligned}
& T_{c}=\iint_{S} \tau_{y} r_{m} d A \\
& T_{\eta}=\iint_{S} \eta \dot{\gamma}_{m} d A
\end{aligned}
$$

where $S$ is the contact area between ER fluid and outer electrode. $r_{m}$ is the moment arm distance from the one point on the inner electrode to rotational axis. In spherical joint mechanism, there is a pitching, rolling, and yawing rotational axis as shown in Fig.2. The moment arms of three rotational axes are expressed as follows:

$$
\begin{aligned}
& { }^{Z} r_{m}=r_{e} \cos v \\
& { }^{X} r_{m}\left(={ }^{Y} r_{m}\right)=r_{e} \sqrt{(\sin v)^{2}+(\cos v \sin u)^{2}}
\end{aligned}
$$

where $r_{e}$ is the radius of inner electrode. Since geometric shape of ER spherical joint along $\mathrm{X}$ and $\mathrm{Y}$ axes is identical with the inner and outer electrodes as shown in Fig.1., moment arms along $\mathrm{X}$ and $\mathrm{Y}$ axes are the same. As shown in Fig.2., an arbitrary point of the ER spherical joint is represented by the angles $u$ and $v$. Under consideration of Eqs. (3)-(4), Eq. (2) can be rewritten as follows:

$$
\begin{gathered}
{ }_{T} T=\int_{-\frac{\pi}{2}}^{v_{o}} \int_{0}^{2 \pi} \tau_{y}(E) r_{e}^{3} \cos ^{2} v d u d v+\int_{-\frac{\pi}{2}}^{v_{o}} \int_{0}^{2 \pi} \eta \frac{\omega}{t_{s}} r_{e}^{4} \cos ^{3} v d u d v \\
\quad{ }^{Z} T_{f} \\
X_{T}\left(={ }^{Y} T\right)={ }^{Z} T_{c,\left(v_{0}=\pi / 2\right)+{ }^{Z} T_{\eta,\left(v_{0}=\pi / 2\right)}{ }^{X} T_{f}} \\
\quad-\int_{0^{2}}^{\frac{\pi}{2}-v_{o}} \int_{0}^{2 \pi} \tau_{y}(E) r_{e}^{3} \sqrt{(\sin v)^{2}+(\cos v \sin u)^{2}} \cos v d u d v \\
-\int_{0^{2}}^{\frac{\pi}{2}-v_{o}} \int_{0}^{2 \pi} \eta \frac{\omega}{t_{s}} r_{e}^{4} \sqrt{(\sin v)^{2}+(\cos v \sin u)^{2}} \cos ^{2} v d u d v
\end{gathered}
$$

where $\omega$ is the angular velocity of the spherical joint, $t_{s}$ is the gap size and $v_{o}$ is constant angle of outer electrode.

From (5), the repulsive torque of ER spherical joint is mainly determined by two design parameters, $r_{e}$ and $v_{o}$. During design procedure, mechanical friction torque is supposed to be zero. The maximum yield stress of the employed ER fluid is $1.26 \mathrm{kPa}$ at $2 \mathrm{kV} / \mathrm{mm}$, and the 
dynamic viscosity constant is $0.53 \mathrm{~Pa} \bullet \mathrm{s}$. In order to achieve the desired magnitude of repulsive torque level such as $0.6 \mathrm{Nm}, r_{e}$ and $v_{o}$ are determined as $41 \mathrm{~mm}$ and 50 degrees, respectively. When angular velocity is assumed to be $1.5 \mathrm{rad} / \mathrm{s}$, the calculated control torques of the $\mathrm{Z}$ axis and the $X, Y$ axes are $0.801 \mathrm{Nm}$ and $0.600 \mathrm{Nm}$, respectively. The friction torques induced by ER fluid along the $\mathrm{Z}$ axis and the $\mathrm{X}, \mathrm{Y}$ axes are $0.0181 \mathrm{Nm}$ and $0.0127 \mathrm{Nm}$, respectively.

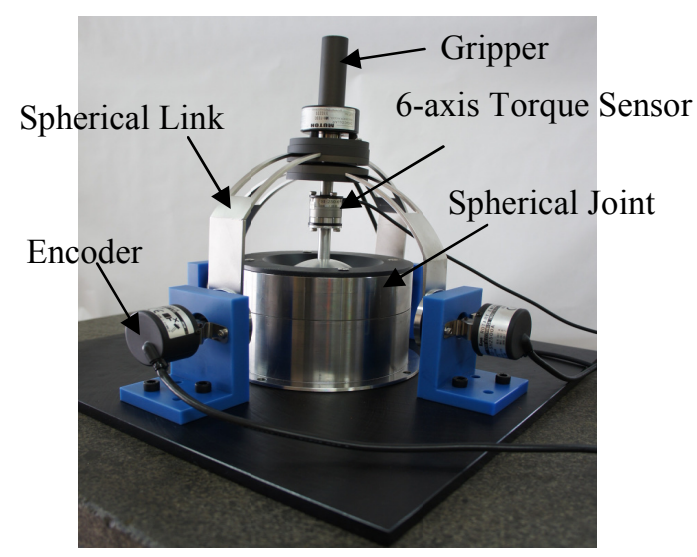

Fig.3. Manufactured ER haptic master.

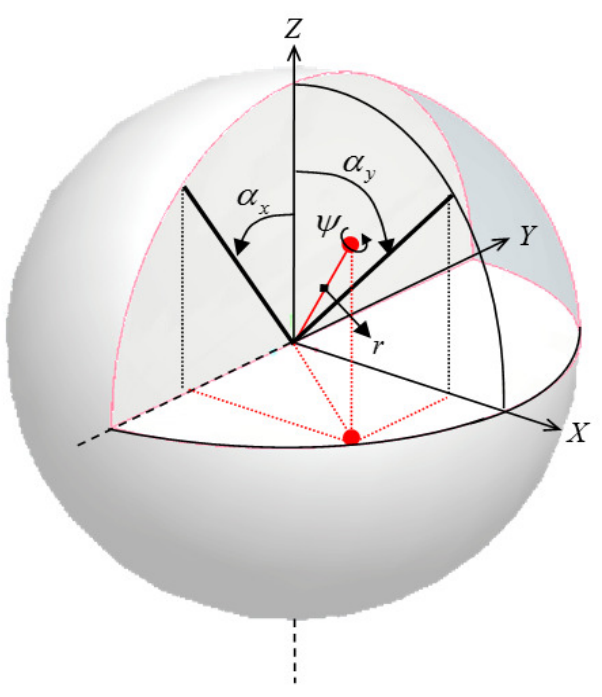

Fig.4. Position measuring mechanism.

With determined design parameters, ER spherical joint was manufactured as shown in Fig.3. In order to measure the generated repulsive torque, 6-axis torque sensor (ATI, Nano 17) is attached at the middle position of gripper connected to the inner electrode. In order to measure rotational position information, spherical link with encoders is connected to the gripper. It is noted that the measured rotational position information is converted to command signal for slave robot in the RMIS system. As shown in Fig.4., the end position of the gripper, determined by pitching angle and rolling angle, can be expressed as follows:

$$
\begin{aligned}
& x=r \cos \alpha_{x} \sin \alpha_{y} \\
& y=r \sin \alpha_{x} \\
& z=r \cos \alpha_{x} \sin \alpha_{y}
\end{aligned}
$$

where $\alpha_{x}$ and $\alpha_{y}$ are measured rotational signals from encoders. $r$ is length between ER spherical joint and spherical link.

\section{TORQUE MEASUREMENT}

In this work, a rotational coaxial cylinder type viscometer is utilized to obtain parameters for the Bingham model. As shown in Fig.5., the viscometer consists of torque sensor, rotating inner cylinder and stationary outer housing. It is also noted that edge effect in the bottom of cylinder is assumed to be zero to simplify the torque and shear rate modeling. It is also remarked here that the edge effect from the above assumption has been observed to be very small in this experiment. The speed of inner cylinder is controlled by a DC servomotor (Mitsubishi Corp., HC-KFS73) to get the shear rate up to $2,000 \mathrm{~s}^{-1}$. The maximum torque and speed of the DC servomotor is $7.2 \mathrm{Nm}$ and $4500 \mathrm{rev} . / \mathrm{min}$, respectively. The gap between the inner cylinder and the outer housing is fixed by $1 \mathrm{~mm}$. The torque signal is measured by the torque sensor (Dacell, TRD-5KC). The maximum input to the viscometer is $2 \mathrm{kV}$. Once the inner cylinder is rotated with constant velocity and constant electric field, torque signal is measured as shown in Fig.6. The electric field is applied to ER fluid for $1 \mathrm{sec}$. After the electric field is applied, the torque value is collected for $0.2 \mathrm{sec}$ and the average value of measured torque is calculated to minimize the overshoot effect of torque value in initial response. From the average torque value, the shear stress, $\tau$, and the shear rate, $\dot{\gamma}$, can be calculated as follows [12]:

$$
\begin{aligned}
& \tau=\frac{T}{2 \pi r^{2} L C_{L}} \\
& \dot{\gamma}=\max \left(\dot{\gamma}_{a} ; \dot{\gamma}_{b}\right) \\
& \dot{\gamma}_{a}=2 T \frac{d \omega}{d T} \\
& \dot{\gamma}_{a}=2 \frac{T(d \omega / d T)}{1-\left(R_{i}^{2} / R_{o}^{2}\right)}-\frac{\omega-T(d \omega / d T)}{\ln \left(R_{i} / R_{o}\right)}
\end{aligned}
$$

where $T$ is the measured torque value and $C_{L}$ is the resistance coefficient of surface correction. The value of $C_{L}$ is normally determined by empirical method and in this work this value is determined by 1.1 [12]. $L$ is the length of the inner cylinder. $R_{o}$ is the radius of the outer cylinder, and $R_{i}$ is the radius of the inner cylinder. For more detailed information, please refer to our previous study [12]. Fig.7. shows the shear stress calculated from the measured torque according to numerous velocity and electric input conditions. From Fig.7., the yield stress of ER fluid is obtained from the intercept at zero shear rates. As shown in Fig.8., the dynamic yield stress of the employed ER fluid is expressed by $500.2 E^{1.584}$. 


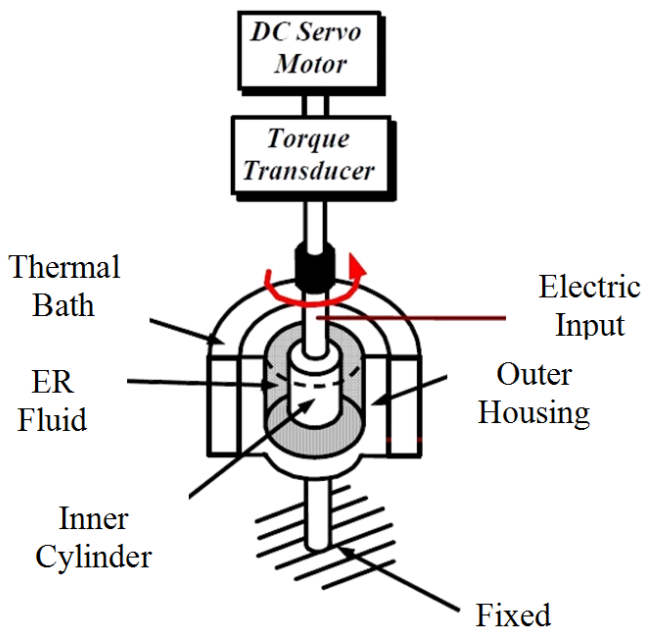

(a) configuration

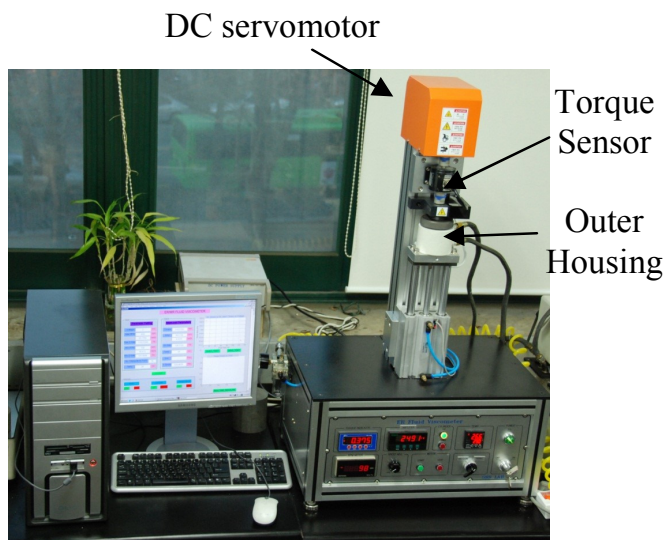

(b) photograph

Fig.5. ER Viscometer.

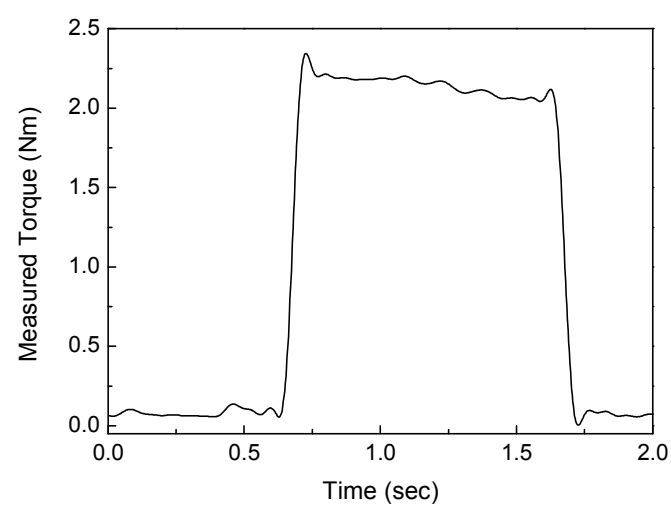

Fig.6. Field-dependent Torque of ER Fluid.

After manufacturing the ER haptic master, the generated repulsive torque performance of the haptic master device is evaluated. Fig.9. shows an experimental apparatus for torque measurement. In order to rotate the gripper with constant angular velocity, the AC motor attached to the spherical link is employed. When the gripper rotates along the rotational axes, torque information is obtained from 6axis torque sensor (ATI Corp., Nano 17), and transferred to the microprocessor (computer). Step input is amplified by high voltage amplifier and transferred to the haptic master.
All signals are converted to digital signal via $\mathrm{A} / \mathrm{D}, \mathrm{D} / \mathrm{A}$ board, which is NI Express Chassis (PXIe-1082) including Data Acquisition Board (PXIe-6363) and Waveform Analog Output Board (PXI-6723).

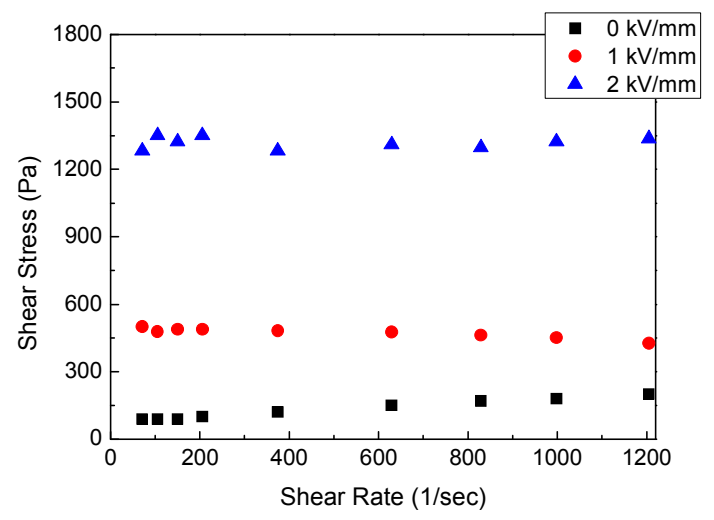

Fig.7. Shear Stress of ER Fluid.

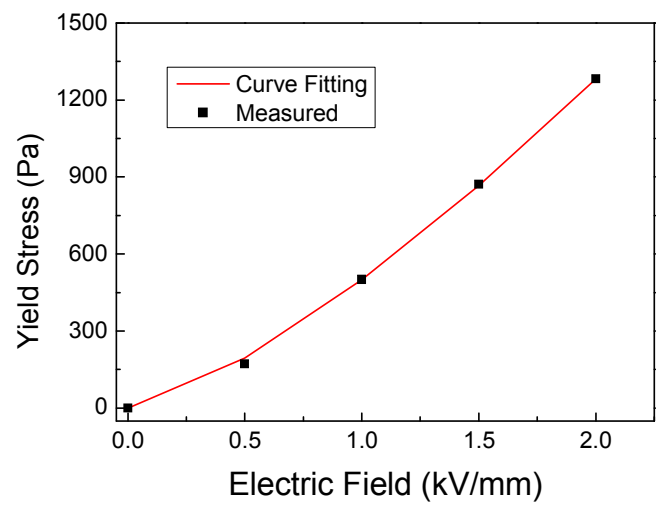

Fig.8. Bingham characteristics of ER fluid.

Table 1. Comparison of motion error

\begin{tabular}{ccc}
\hline $\begin{array}{c}\text { Input } \\
\text { Condition }\end{array}$ & $\begin{array}{c}\text { Error for Yawing } \\
\text { Motion }\end{array}$ & $\begin{array}{c}\text { Error for Pitching \& } \\
\text { Rolling Motions }\end{array}$ \\
\hline \hline $0.5 \mathrm{kV}$ & $0.005 \mathrm{Nm}$ & $0.003 \mathrm{Nm}$ \\
\hline $1 \mathrm{kV}$ & $0.021 \mathrm{Nm}$ & $0.011 \mathrm{Nm}$ \\
\hline $1.5 \mathrm{kV}$ & $0.016 \mathrm{Nm}$ & $0.001 \mathrm{Nm}$ \\
\hline $2 \mathrm{kV}$ & $0.039 \mathrm{Nm}$ & $0.044 \mathrm{Nm}$ \\
\hline
\end{tabular}

Fig.10. presents the repulsive torque measured by the 6 axis torque sensor and the simulation results calculated by utilizing (5). The maximum generated torques are $0.761 \mathrm{Nm}$ (yawing motion) and $0.559 \mathrm{Nm}$ (rolling motion) at $2 \mathrm{kV} / \mathrm{mm}$, respectively. From the experimental results, the proposed haptic master device can provide appropriate torque level for the force-feedback performance to the operator without exhibiting significant errors between simulation value and experimental result. In addition, Table 1. shows the difference value between measured torque results and simulation results at a steady state. The maximum error value and average error percentage are evaluated by $0.044 \mathrm{Nm}$ and $4.73 \%$, respectively. From 
these results, it is demonstrated that the predicted performance is successfully achieved by implementing the proposed 3-DOF haptic master. Additionally, torque tendency of $\mathrm{Z}$ axis behaves as an underdamped system, while torque tendency of $\mathrm{X}$ and $\mathrm{Y}$ axes behaves as an overdamped system. Thus, the second-order dynamic models along 3-DOF rotational motions can be derived as follows:

$$
\begin{aligned}
& \ddot{F}_{i}+2 \xi_{i} \omega_{n, i} \dot{F}_{i}+\omega_{n, i}^{2} \quad F_{i}=\omega_{n, i}^{2} I_{i} u_{i}, \quad i=1,2 \\
& \ddot{T}+2 \xi_{3} \omega_{n, 3} \dot{T}+\omega_{n, 3}^{2} T=\omega_{n, 3}^{2} I_{3} u_{3}
\end{aligned}
$$

where $u_{i}$ is the generalized force, $F_{i}$ is the produced force induced by repulsive torque of pitching and rolling motions at the gripper, and $T$ is the produced torque in yawing motion. $I_{i}$ is the influence coefficient due to the generated torque/force per unit yield stress. And $\xi_{i}$ is damping ratio and $\omega_{n, i}$ is natural frequency of the dynamic models.
The subscript numbers such as 1, 2 and 3 are used to distinguish the three different rotational motions. The detailed information for dynamic models is listed in Table 2.

Table 2. Dynamic properties of ER haptic master

\begin{tabular}{cc}
\hline Parameter & Value \\
\hline \hline$\xi_{1}$ & 0.603 \\
\hline$\omega_{n, 1}$ & $29.38 \mathrm{rad} / \mathrm{sec}$ \\
\hline$\xi_{2}$ & 0.993 \\
\hline$\omega_{n, 2}$ & $23.98 \mathrm{rad} / \mathrm{sec}$ \\
\hline$\xi_{3}$ & 0.948 \\
\hline$\omega_{n, 3}$ & $8.63 \mathrm{rad} / \mathrm{sec}$ \\
\hline
\end{tabular}

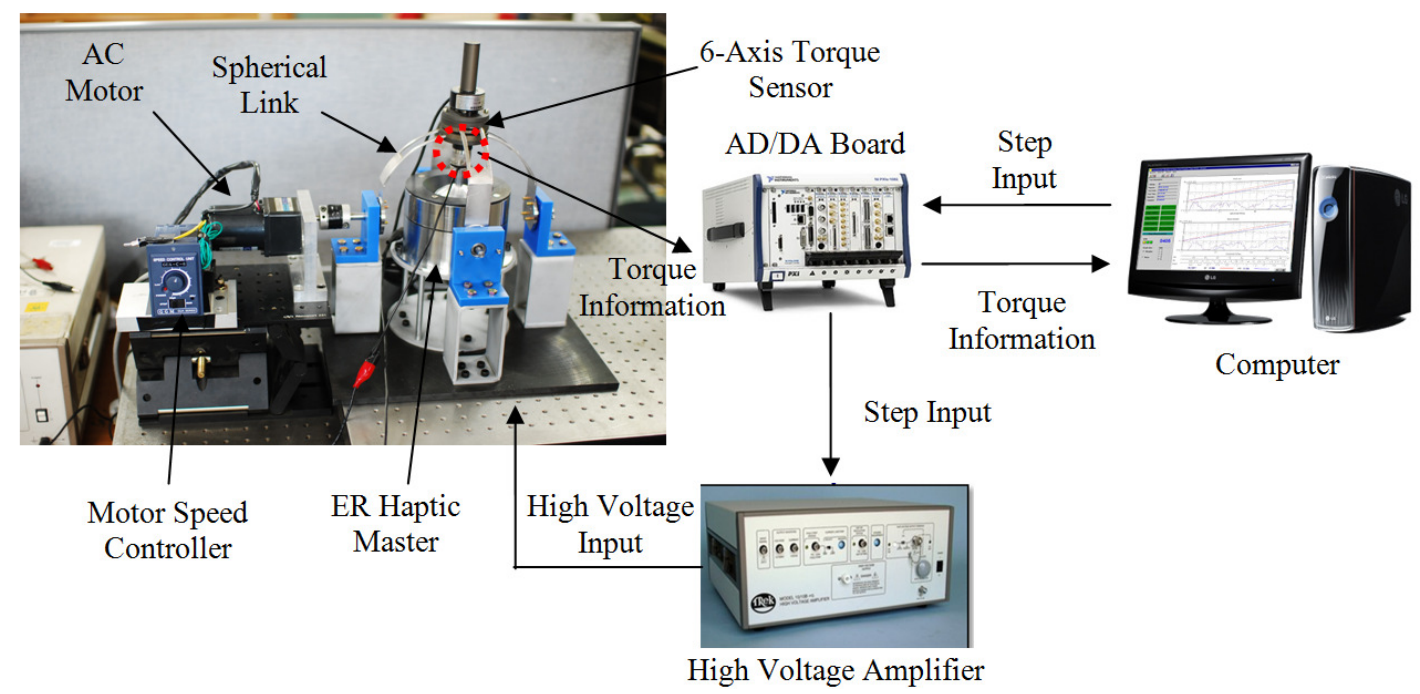

Fig.9. Experimental apparatus for torque measurement of ER haptic master.

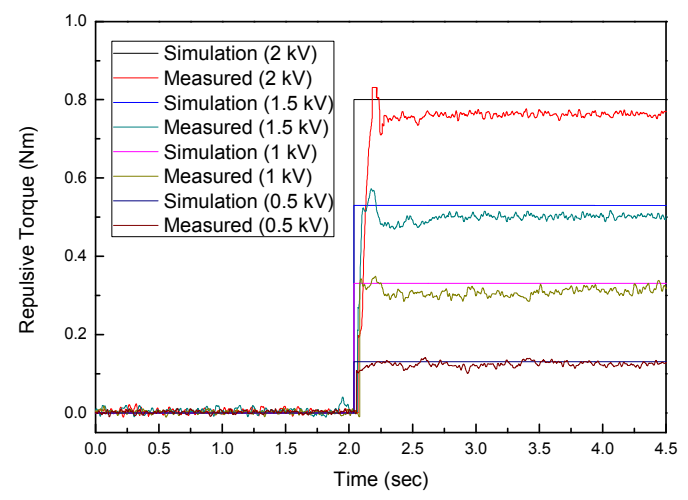

(a) generated torque result for $\mathrm{Z}$ axis, ${ }^{Z} T$

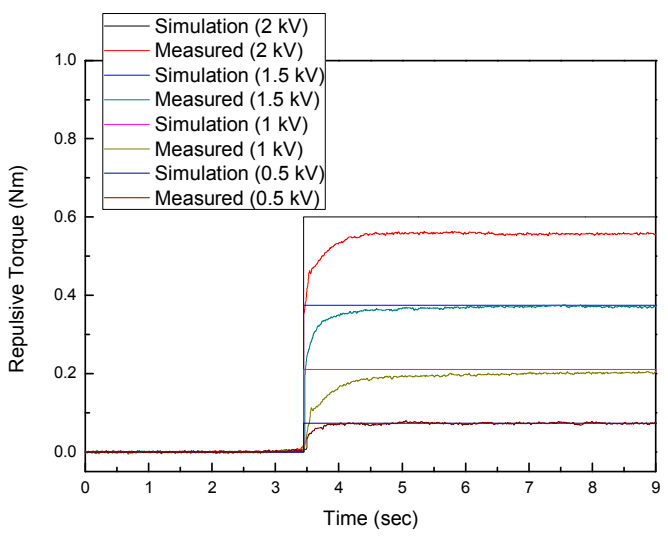

(b) generated torque results for $\mathrm{X}$ and $\mathrm{Y}$ axes, ${ }^{X} T\left(={ }^{Y} T\right)$

Fig.10. Generated torque of ER haptic master. 


\section{CONCLUSION}

In this work, a repulsive torque measurement generated from 3-DOF ER haptic master featuring spherical joint mechanism was conducted. By controlling the electric field applied to ER fluid, the haptic device can easily generate repulsive torque along the 3-DOF directions. After demonstrating the mechanical configuration of the proposed haptic master device, the torque models of the proposed haptic master were mathematically derived based on the Bingham model of ER fluid. In order to verify the validity of the proposed torque model, the yield stress performance of ER fluid and the torque performance of manufactured haptic device were measured and evaluated. The experiment results show that the torque model for the proposed haptic master can generate the measured torque levels without significant errors. It has been also shown from the measured torque levels that the proposed haptic master can be effectively utilized for the repulsive torque required to operate the robot-assisted surgery application.

\section{ACKNOWLEDGEMENT}

This work was supported by a National Research Foundation of Korea (NRF) grant funded by the Korea government (MEST) (No. 2010-0015090). This financial support is gratefully acknowledged.

\section{REFERENCES}

[1] Stanway, R., Sporoson, J.L. (1994). Electrorheological fluid: A systematic approach to classifying modes of operation. Journal of Dynamic Systems, Measurement, and Control, 116, 498-504.

[2] Lee, H.G., Choi, S.B., Han, S.S., Kim, J.H., Suh, M.S. (2001). Bingham and response characteristics of ER fluids in shear and flow modes. Intelligent Journal of Modern Physics B, 15, 1017-1024.

[3] Sung, K.G., Seong, M.S., Choi, S.B. (2012). Performance evaluation of electronic control suspension featuring vehicle ER dampers. Meccanica, 48 (1), 121-134.

[4] Yamaguchi, H., Zhang, X.-R., Niu, X.-D. (2010). Damping characteristics and flow behaviors of an ER fluid with a piston sine vibration in a viscous damper. Smart Materials \& Structures, 19 (10), 105032.
[5] Tan, K.P., Stanway, R., Bullough, W.A., (2006) Robot arm control using an electro-rheological (ER) clutch-brake mechanism: Model validation. International Journal of Modern Physics B, 20 (2), 181-216.

[6] Yook, J.Y., Choi, S.B., Yook, W.S. (2012). Design and speed control of ER brake system using GER fluids. Transactions of the Korean Society for Noise and Vibration Engineering, 22 (4), 365-371.

[7] Pierrot, F., Dombre, E., Dégoulange, E., Urbain, L., Caron, P., Sylvie, B., Gariépy, J., Mégnien, J. (1999). Hippocrate: A safe robot arm for medical applications with force feedback. Medical Image Analysis, 3 (3), 285-300.

[8] Kikuchi, T., Fukushima, K., Furusho, J. (2009). Development of Quasi-3DOF upper limb rehabilitation system using ER brake: PLEMO-P1. Journal of Physics: Conference series, 149 (1), 012015.

[9] Furusho, J., Sakaguchi, M., Takesue, N. (2002). Development of ER brake and its application to passive force display. Journal of Intelligent Material Systems and Structures, 13 (7/8), 425-430.

[10] Choi, S.B., Lee, D.Y. (2005). Rotational motion control of a washing machine using electrorheological clutches and brakes. Proceedings of the Institution of Mechanical Engineers, Part C: Mechanical Engineering Science, 219 (7), 627-638.

[11] Han, Y.M., Kim, C.J., Choi, S.B. (2009). A magnetorheological fluid-based multifunctional haptic device for vehicular instrument controls. Smart Materials \& Structures, 18 (1), 015002.

[12] Choi, S.B., Han, Y.M., Sohn, J.W., Choi, H.J. (2009). Bingham characteristics of polymer-based electrorheological fluids with different electrode gaps and materials. Journal of Applied Polymer Science, 114 (6), 3636-3644.

[13] Estellé, P., Lanos, P., Perrot, A. (2006). Processing the Couette viscometry using a Bingham approximation in shear rate calculation. Journal of Non-Newtonian Fluid Mechanics, 154 (31-38), 31-3.

Received May 15, 2014. Accepted January 14, 2015. 